\title{
- The Sacred Path of the Therapist: Modern Healing, Ancient Wisdom, and Client Transformation
}

\author{
Irene R. Siegel. New York, NY: W. W. Norton \& Company, 2017, 215 pages, \$27.50 (Hardcover), \$15.12 (Kindle)
}

The field of transpersonal psychology recognizes the spiritual dimensions and levels of consciousness depicted in ancient wisdom traditions. Dr. Siegel's book teaches therapists how to awaken their own spiritual consciousness and bring this awareness into the psychotherapeutic process so that they can become an "instrument of transformation." She provides a series of meditations and numerous exercises to guide the therapist step by step toward greater inner awareness. She teaches the skills of alternative ways of knowing, tracking information and energy flow, and how to hold a resonance in a shared energy field - based in connection to a divine source-in the therapy session. Detailed descriptions explain the therapeutic process through the overarching concept and felt experience of spiritual resonance.

The author's own spiritual lineage, via Alberto Villoldo, is key. Having apprenticed in the shamanic tradition in Peru with indigenous master teachers of Incan descent, she exemplifies the development of consciousness that her book promotes. Her practice also incorporates Eastern traditions, embodying ancient wisdom and referencing current practices. The book is well researched, and recent scientific findings illuminate spiritual teachings.

The book serves as the integration of a transpersonal approach into the eye movement desensitization and reprocessing (EMDR) protocol that one can adapt into one's own therapy practice, thereby opening the door to deeper consciousness and perhaps transcendence. It provides instruction on how to incorporate transpersonal elements within each EMDR treatment phase. For example, Dr. Siegel explains how bilateral stimulation (BLS) can be used with clients to foster a shared experience of an expanded energy field. She departs from the standard EMDR protocol of observing past trauma and present stimuli at the same time from an egoic perception with open eyes. Instead, the client closes her eyes while processing, wearing headphones with auditory BLS - in resonance with divine presence as a catalyst for transformation. The book is richly illustrated with client cases. These are highly instructive, and the clients' major shifts of consciousness are reported within the standard EMDR protocol, with descriptions of negative and positive cognitions, subjective units of disturbance (SUD) scores, BLS processes, and cosmic interweaves (yes, cosmic interweaves). Clients present with a range of issues, with a few cases of physical healing.

I was most impressed with the case of a Hispanic woman recovering from a traumatic motorcycle accident involving the death of her boyfriend. Teresa's therapy was not just about healing from posttraumatic stress. Spontaneously, without any spiritual perspective, a spiritual awakening unfolded as Teresa began to experience her spiritual essence as an integral part of her own identity. Her case answered my concern that this transpersonal psychotherapy would only be appropriate for clients who already self-identified with a spiritual perspective.

Dr. Siegel assures us that mystical, peak, and extraordinary human experiences (EHEs) can be observed within the therapeutic container; she is thereby an adherent to the fifth force of psychology, where spiritual resonance is a core component of psychotherapy. The Sacred Path of the Therapist is a significant contribution that I highly recommend for any level of clinician.

REVIEWED BY JANET ELIZABETH COLLI 